\title{
GENERALIZATIONS OF ACCRETIVITY FOR NONLINEAR MAPPINGS OF BANACH SPACES ${ }^{1}$
}

\author{
BY FELIX E. BROWDER
}

Communicated August 2, 1971

Let $X$ and $Y$ be real Banach spaces, $f$ a mapping of $X$ into $2^{Y}, y_{0}$ an element of $Y$. It is our object in the present discussion to present a new existence theory for solutions of the equation $y_{0} \in f(x)$ which extends, sharpens, and simplifies the corresponding theory for $X=Y$ and $f$ hypermaximal accretive. This new existence theory is founded in a very basic way upon the fixed point theory of self-mappings of the Banach space $Y$ (for example, of mappings of nonexpansive or of condensive type) and yields in its turn an interesting extension of these fixed point theories.

In its most general form, the class of mappings which we wish to treat is given in the following form:

Definition 1. Let $X$ and $Y$ be Banach spaces, $K$ a mapping of $X$ into $Y$, $F$ a class of mappings of $Y$ into $2^{Y}$.

The mapping $f$ of $X$ into $2^{Y}$ is said to $(F, K)$-generative if the mapping $g=K(f+K)^{-1}$ lies in the class $F$. If $X=Y$ and $K$ is the identity mapping, we say that $f$ is $F$-generative. If, in addition, $F$ is the class of nonexpansive mappings of $Y$ into $Y$, we say that $f$ is generative.

We recall that a mapping $f$ of $Y$ into $2^{Y}$ is said to be hypermaximal accretive ( $m$-accretive) if for each $\xi>0,(I+\xi f)^{-1}$ is a nonexpansive mapping of $Y[\mathbf{1}]-[\mathbf{5}]$. In terms of Definition $1, f$ is hypermaximal accretive if $\xi f$ is generative for each $\xi>0$. If $Y$ is a Hilbert space and the range of $(I+f)$ is all of $Y$, then $f$ is hypermaximal accretive whenever

$$
(y-w, x-u) \geqq 0
$$

for all $y$ in $f(x), w$ in $f(u)$, while $f$ is generative if the following weaker inequality is satisfied

$$
(y-w, x-u) \geqq-\frac{1}{2}\|y-w\|^{2} .
$$

In our results, we impose several conditions upon the class of mappings $F$ which are described in detail in the following definition:

DEFINITION 2.F is said to be closed under translation if for each $g$ in $F$ and each pair of elements $w_{0}$ and $w_{1}$ of $Y$, the mapping $g_{0}$ given by $g_{0}(y)=$ $g\left(y+w_{0}\right)+w_{1}$ also lies in the class $F$.

AMS 1970 subject classifications. Primary 47H15, 47H10; Secondary 47H05.

${ }^{1}$ Supported by NSF GP-28148. 
$F$ is said to have the weak fixed point property on balls if for each $R_{0}>0$ and each $g$ in $F$ such that, for each $R \geqq R_{0}, g$ maps the ball $B_{R}(0, Y)$ into itself, there exists a fixed point $w_{0}$ of $g$ in $B_{R_{0}}(0, Y)$, i.e. $w_{0} \in g\left(w_{0}\right)$.

$F$ is said to have the weak almost-fixed point property on balls if for each $R_{0}>0$ and each $g$ in $F$ such that for all $R \geqq R_{0}, g$ maps $B_{R}(0, Y)$ into itself, then $g$ has the almost-fixed point property on $B_{R_{0}}(0, Y)$, i.e. 0 lies in the closure of $(I-g)\left(B_{R_{0}}(0, Y)\right)$.

For stating our basic results, we also need the following generalization of the concept of duality mapping and of coercivity.

Definition 3. Let $K$ be a mapping of $X$ into $Y$. Then a mapping $M$ of $X$ into $Y^{*}$ is said to be a duality mapping for $K$ if, for each $u$ in $X$,

$$
(M(u), K(u))=\|K(u)\|^{2}, \quad\|M(u)\|=\|K(u)\| .
$$

The mapping $f$ of $X$ into $2^{Y}$ is said to be coercive with respect to the mapping $M$ if there exists a function $c$ from $R^{+}$to $R^{1}$ such that $\lim _{r \rightarrow \infty} c(r)$ $=+\infty$ such that for each $u$ in $X$ and each $y$ in $f(u)$,

$$
(y, M(u)) \geqq c(\|u\|)\|M(u)\| .
$$

Our basic existence theorems are the following:

THEOREM 1. Let $X$ and $Y$ be Banach spaces, $f$ a mapping of $X$ into $2^{Y}, y_{0} a$ point of $Y$. Let $K$ be a bounded mapping of $X$ into $Y, M$ a mapping of $X$ into $Y^{*}$ which is a duality mapping for $K, F$ a translation invariant class of mappings of $Y$ into $2^{Y}$ such that $F$ has the weak fixed point property on balls. Suppose that $f$ is $(F, K)$-generative, and that there exists $R_{0}>0$ such that for each $u$ in $X$ with $\|u\| \geqq R_{0}$, we have the inequality

$$
(y, M(u)) \geqq\left(y_{0}, M(u)\right)
$$

for each $y$ in $f(u)$.

Then there exists $u_{0}$ in $X$ such that $y_{0} \in f\left(u_{0}\right)$.

Corollary 1 to Theorem 1. If, in Theorem 1, we either have $X=Y$ with $K$ the identity mapping or replace (1) by the strict inequality

$$
(y, M(u))>\left(y_{0}, M(u)\right)
$$

for $\|u\|>R_{0}, y \in f(u)$, then the solution $u_{0}$ of the equation $y_{0} \in f\left(u_{0}\right)$ can be chosen in $B_{R_{0}}(0, X)$.

COROLlaRy 2 TO THEOREM 1. If, in Theorem 1, we replace the inequality (1) by the hypothesis that $f$ is coercive with respect to $M$, then the range of $f$ is all of $Y$.

THEOREM 2. Suppose that the hypotheses of Theorem 1 all hold except that $F$ satisfies the weak almost-fixed point property on balls rather than the weak 
fixed point property on balls. We then obtain the weaker conclusion that $y_{0}$ lies in the closure of the range of $f$. If either $X=Y$ with $K$ the identity map or the strict inequality (2) holds, then $y_{0}$ lies in the closure of $f\left(B_{R_{0}}(0, X)\right)$.

Corollary to Theorem 2. If $F$ satisfies the weak almost-fixed point property on balls, $f$ is a mapping of $X$ into $2^{Y}$ which is $(F, K)$-generative and with $f$ coercive with respect to $M$, then the range of $f$ is dense in $Y$.

To give a particular application of Theorems 1 and 2, we apply them to the special case of generative mappings.

THEOREM 3. Let $Y$ be a Banach space, $f$ a coercive generative mapping of $Y$ into $2^{Y}$ (and in particular, $f$ may be hypermaximal accretive). Then the range of $f$ is dense in $Y$. If closed balls in $Y$ have normal structure in the sense of Brodski and Milman, the range of $f$ is all of $Y$.

For the case in which both $Y$ and $Y^{*}$ are uniformly convex, the result of Theorem 3 was proved by the writer in [2], [3] for hypermaximal accretive $f$. H. Brezis pointed out in a letter to the writer that a modification of the argument in [3] yields the conclusion without assuming that $Y^{*}$ is uniformly convex (which was the stimulus to the study given in the present paper). For general $Y$ and $f$ hypermaximal accretive and coercive, the density of the range of $f$ has been noted independently by Yen in a recent note $[8]$.

ProOF OF TheOREM 1. Let $y_{0}$ be the given point of $Y$. By hypothesis, if $g=K(f+K)^{-1}$, the mapping $g$ lies in the class $F$. Let $f_{0}$ be the mapping of $X$ into $2^{Y}$ given by $f_{0}(y)=f(y)-y_{0}$. A given element $w$ of $Y$ lies in $\left(f_{0}+K\right)(x)$ for a point $x$ in $X$ if and only if $\left(w+y_{0}\right) \in(f+K)(x)$, i.e.

$$
\left(f_{0}+K\right)^{-1}(w)=(f+K)^{-1}\left(w+y_{0}\right) .
$$

Since $g=K(f+K)^{-1}$ is a member of the class $F$, and since $K\left(f_{0}+K\right)^{-1}(w)=g\left(w+y_{0}\right)$, it follows from the assumption that $F$ is translation invariant that the mapping $g_{0}=K\left(f_{0}+K\right)^{-1}$ lies in the class $F$.

Since $K$ is assumed to be a bounded mapping (i.e. $K$ maps bounded sets of $X$ into bounded subsets of $Y)$, there exists $R_{1}>0$ such that $K\left(B_{R_{0}}(0, X)\right)$ $\subset B_{R_{1}}(0, Y)$. (When $X=Y$ and $K$ is the identity mapping, we take $R_{1}=R_{0}$.) We now assert that for each $R \geqq R_{1}$, the mapping $g_{0}$ carries $B_{R}(0, Y)$ into itself. Indeed, let $w$ be an element of $B_{R}(0, Y)$ for such an $R$, and let $y$ be a point of $g_{0}(w)$ such that $\|y\| \geqq R$. Then $y=K(x)$ for a point $x$ in $X$ such that $x \in\left(f_{0}+K\right)^{-1}(w)$, i.e. $w \in f_{0}(x)+K(x)$. Thus there exists an element $v$ of $f(x)$ such that $w=v-y_{0}+K(x)$. Since $y$ lies outside of $B_{R_{1}}(0, Y)$, and $y=K(x)$, it follows that $x$ lies outside of $B_{R_{0}}(0, X)$. Hence the inequality (1) 
of the hypothesis of Theorem 1 implies that $\left(M(x), v-y_{0}\right) \geqq 0$. Since $M$ is a duality mapping for $K$, we have

$$
(M(x), K(x))=\|K(x)\|^{2}=\|y\|^{2} .
$$

Hence

$$
(M(x), w)=\left(M(x), v-y_{0}\right)+(M(x), K(x)) \geqq\|y\|^{2},
$$

so that

$$
\|y\|^{2} \leqq\|M(x)\| \cdot\|w\|=\|K(x)\| \cdot\|w\|=\|y\| \cdot\|w\| .
$$

Finally, we see that $\|y\| \leqq\|w\| \leqq R$.

By hypothesis, $F$ has the weak fixed point property on balls. Hence $g_{0}$ has a fixed point $w_{0}$ in $B_{R_{1}}(0, Y)$ such that $w_{0} \in g_{0}\left(w_{0}\right)$. For such an element $w_{0}$, we have $w_{0}=K\left(x_{0}\right)$ while $w_{0} \in f\left(x_{0}\right)-y_{0}+K\left(x_{0}\right)$. It follows that $y_{0} \in f\left(x_{0}\right)$. q.e.d.

Proof of Corollary 1 to Theorem 1 . If $X=Y$ and $K$ is the identity, then $w_{0}=x_{0}$, and by our choice of $R_{1}, R_{1}=R_{0}$. Therefore, $y_{0}$ lies in $f\left(B_{R_{0}}(0, X)\right.$ ). If the inequality (2) holds (with a strict inequality), it excludes $y_{0} \in f\left(x_{0}\right)$ if $x_{0}$ lies outside the ball $B_{R_{0}}(0, X)$. q.e.d.

ProOF OF COROLlaRY 2 TO TheOREM 1. The coerciveness of $f$ with respect to $M$ implies trivially that the inequality (1) holds for a given $y_{0}$ of $Y$ outside of some ball $B_{R_{0}}(0, X)$ depending upon $y_{0}$. Hence by Theorem $1, R(f)$ $=Y$. q.e.d.

ProOF OF THEOREM 2. We apply the proof of Theorem 1 and obtain the conclusion that $g_{0}$ maps $B_{R}(0, Y)$ into itself for each $R \geqq R_{1}$. If $F$ has the weak almost-fixed point property on balls, it follows that 0 lies in the closure of $\left(I-g_{0}\right)\left(B_{R_{1}}(0, Y)\right)$. Thus, there must exist a sequence $\left\{w_{n}\right\}$ in $B_{R_{1}}(0, Y)$ and for each $n$, an element $y_{n}$ of $g_{0}\left(w_{n}\right)$ such that $w_{n}-y_{n} \rightarrow 0$ as $n \rightarrow \infty$. Since $y_{n} \in g_{0}\left(w_{n}\right)$, there exists $x_{n}$ in $X$ for each $n$ such that $y_{n}=K\left(x_{n}\right)$ while $x_{n} \in\left(f_{0}+K\right)^{-1}\left(w_{n}\right)$. Hence, there exists $v_{n}$ in $f\left(x_{n}\right)$ such that

$$
w_{n}=v_{n}-y_{0}+K\left(x_{n}\right)=y_{n}+v_{n}-y_{0} .
$$

Hence, $v_{n}-y_{0}=w_{n}-y_{n} \rightarrow 0$ as $n \rightarrow \infty$, and $y_{0}$ lies in the closure of the range of $f$. Under the appropriate additional hypotheses, $y_{0}$ lies in the closure of $f\left(B_{R_{0}}(0, X)\right)$. q.e.d.

The proof of the Corollary to Theorem 2 follows like that of Corollary 2 to Theorem 1.

The proof of Theorem 3 follows directly from Theorems 1 and 2 applied to the case of the class $F_{0}$ of nonexpansive mappings of $Y$, since $F_{0}$ has the weak almost-fixed point property on balls for any Banach space $Y$ and the weak fixed point property if $Y$ satisfies the condition that closed balls have normal structure. 
THEOREM 4. The results of Theorems 1,2 , and 3 as well as their Corollaries all remain valid if the hypothesis that $f$ is $(F, K)$-generative is replaced by the hypothesis that there exists $\alpha>0$ such that $\alpha f$ is $(F, K)$-generative.

We merely note that $y_{0} \in(\alpha f)\left(x_{0}\right)$ if and only if $\alpha^{-1} y_{0} \in f\left(x_{0}\right)$, while the inequality (1) goes over from $f$ to $\alpha f$ in the appropriate way.

REMARK. An interesting specialization of the above results for $X=Y$ and $K$ the identity mapping is that in which we take for the class $F$, the class $F_{c}$ of condensing mappings in the sense of Sadovski [7] to which Theorem 1 may be applied.

The construction of the class of $(F, K)$-generative mappings from a class $F$ of mappings such that solvability properties of the equation $y_{0} \in f\left(x_{0}\right)$ for the generative mapping $f$ follow from the fixed point properties of the mapping $g=K(f+K)^{-1}$ in $F$ has an inverse process by which we generate classes of mappings having the weak fixed point property on balls from $F$-generative mappings.

THEOREM 5. Let $Y$ be a Banach space, $F$ a translation invariant class of mappings of $Y$ into $2^{Y}$. We generate two sequences of classes of mappings $\left\{F_{n}\right\}$ and $\left\{G_{n}\right\}(n \geqq 1)$ by the following recursive definition:

For $n=0$, let $F_{0}=F$. For $n \geqq 1, G_{n}=\left\{f \mid f: Y \rightarrow 2^{Y}\right.$, there exists $\alpha>0$ such that $(I+\alpha f)^{-1}$ lies in $\left.F_{n-1}\right\}$. For $n \geqq 1, F_{n}=\left\{g \mid g: Y \rightarrow 2^{Y}, g=I-f\right.$ for some $f$ in $G_{n}$ \}.

Then if $F$ has the weak fixed point property on balls, so does each $F_{n}$, and each mapping $f$ in $G_{n}$ satisfies the conditions of Theorem 1.

If $F$ has the weak almost-fixed point property on balls, so does each $F_{n}$, and each mapping $f$ in any $G_{n}$ satisfies the condition of Theorem 2.

PROOF OF THEOREM 5. It follows by a routine verification that each of the classes $F_{n}$ and $G_{n}$ can be recursively proved to be translation invariant. Suppose $F_{n-1}$ has been shown to have the weak fixed point property. Then each mapping $f$ in $G_{n}$ satisfies the conclusions of Theorem 1. We now show that $F_{n}$ satisfies the weak fixed point property on balls. By definition, if $g$ is an element of $F_{n}, g=I-f$ for some $f$ in $G_{n}$. Suppose that there exists $R_{0}>0$ such that for each $R \geqq R_{0}, g$ maps $B_{R}(0, Y)$ into $B_{R}(0, Y)$. Let $u$ be an element outside of $B_{R_{0}}(0, Y), R=\|u\|, y \in f(u)$. Then

$$
(y, M(u))=(u, M(u))+(y-u, M(u)) \geqq R^{2}-\|y-u\| R \geqq 0
$$

where $M$ is a section of the duality mapping $J$, since $u-y \in g(u)$ implies that $\|u-y\| \leqq R$. Hence by Theorem 1 and Corollary 1 , there exists an element $u_{0}$ of $B_{R_{0}}(0, Y)$ such that $0 \in f\left(u_{0}\right)$, i.e. $u_{0} \in g\left(u_{0}\right)$. Hence $F_{n}$ has the weak fixed point property on balls.

A similar argument for the weak almost-fixed point property follows from Theorem 2. The remainder of Theorem 5 then follows from Theorems 1 and 2. 
REMARK. In the special cases where $F$ is either $F_{0}$, the class of nonexpansive self-mappings of $Y$ into $Y$, or $F_{c}$, the class of condensive self-mappings of $Y$ into $Y$, it is easy to verify that the corresponding classes $F_{0, n}$ and $F_{c, n}$ increase with $n$ and yield interesting classes of self-mappings of $Y$ with the weak fixed point and almost-fixed point properties under the appropriate hypotheses.

REMARK. It follows from a recent result of R. H. Martin [6] that a mapping $f$ of the Banach space $Y$ into itself which is continuous and such that $(I+\xi f)^{-1}$ is nonexpansive on $R(I+\xi f)$ for each $\xi>0$ has the property that $f$ is hypermaximal accretive. This provides an interesting class of maps $f$ for which the result of Theorem 3 applies.

\section{BIBLIOGRAPHY}

1. F. E. Browder, Nonlinear equations of evolution and nonlinear accretive operators in Banach spaces, Bull. Amer. Math. Soc. 73 (1967), 867-874. MR 38 \# 580.

2. - Nonlinear mappings of nonexpansive and accretive type in Banach spaces, Bull. Amer. Math. Soc. 73 (1967), 875-882. MR 38 \# 581.

3. - Nonlinear operators and nonlinear equations of evolution in Banach spaces, Proc. Sympos. Pure Math., vol. 18, part 2, Amer. Math. Soc., Providence, R.I. (to appear).

4. T. Kato, Nonlinear semigroups and evolution equations, J. Math. Soc. Japan 19 (1967), 508-520. MR 37 \# 1820.

5. - Accretive operators and nonlinear evolution equations in Banach spaces, Proc. Sympos. Pure Math., vol. 18, part 1, Amer. Math. Soc., Providence, R.I., 1970, pp. 138-161.

6. R. H. Martin, Jr., A global existence theorem for autonomous differential equations in a Banach space (to appear).

7. B. N. Sadovski1̌, On a fixed point principle, Funkcional. Anal. i Priložen. 1 (1967), no. 2, 74-76. (Russian) MR 35 \#2184.

8. C.-L. Yen, The range of $m$-dissipative sets, Bull. Amer. Math. Soc. (to appear).

Department of Mathematics, University of Chicago, Chicago, Illinois 60637 